\title{
Selecting Reference Signatures for On-Line Signature Verification
}

\author{
G.Congedo, G.Dimauro, A.M. Forte, S.Impedovo, G.Pirlo \\ Dipartimento di Informatica - Università di Bari \\ Via Amendola 173, 70123 Bari, Italy
}

\begin{abstract}
Personal stability in signing in one of the key factors for highaccuracy signature verification. In this paper the local stability of a dynamic signature is measured. The acccuracy of an on-line signature verification system is improved by selecting, among a set of specimens available, the subset of reference signatures which is near-optimal for different signature representation domains in terms of impostor-acceptance error rate.
\end{abstract}

\section{Introduction}

Signature is the costumary way of identifying an individual in our society and it is rightly considered as one of the best means for personal identification; in fact signature cannot be lost, stolen or forgotten. Because of this, with the diffusion of remote data-banks and widely distributed computer networks there is a growing need of effective systems for automatic signature verification as the renewed interest in this field demonstrates [1,2].

Up to now, a lot of efforts have been carried out by many research groups in order to develop high-accuracy systems for automatic signature verification but it is now generally accepted that the performances of such systems can vary strongly depending on several factors. It is quite obvious that a short, commonly-written signature should be more easy to imitate than a long specimen with many pictorial strokes. Recently an attempt has been proposed to estimate quantitatively and apriori the difficulty in reproducing the signature using a functional model of a typical forger [3]. Unfortunately, signature more than other kinds of writings is written from habits and therefore is not possible to modify it in order to obtain higher level of accuracy in automatic verification. Conversely, the detection of stable regions in the signatures of a writer allows to tune the system according to personal characteristics and specific application requirements [4].

In this paper, the local stability in on-line signatures is measured. From the analysis of high/low-stability regions in the signatures of a writer, the near-optimal subset of reference signatures in terms of low impostor acceptance error rate is selected for different representation domains. 


\section{Local Stability in On-Line Signatures.}

Local stability in an on-line signature can be computed in automatic way by an iterative procedure based on multiple matchings among the specimens in the set of genuine data [4]. Let be

$$
\mathrm{S}^{g}: \mathrm{z}^{g}(\mathrm{i})=\left(\mathrm{x}^{g}(\mathrm{i}), \mathrm{y}^{\mathrm{g}}(\mathrm{i})\right) \quad \mathrm{g}=1,2, \ldots, \mathrm{n} ; \mathrm{i}=1,2, \ldots, \mathrm{M}^{\mathrm{g}}
$$

the set of $n$ on-line genuine signatures of a writer, where the coordinates $x^{g}$ (i) and $y^{g}$ (i) describe the position of the pen with respect to the plane of the graphic tablet at time $i$ of the writing process of the $g$-th signature. A normalization process in the space domain, allows to have for each signature $\mathrm{S}^{g}$ :

$$
0 \leq \mathrm{x}_{i}^{g} \leq 1 \quad \text { and } \quad 0 \leq \mathrm{y}_{i}^{g} \leq 1 \quad \text { for } \mathrm{i}=1,2, \ldots, \mathrm{M}^{g}
$$

In order to evaluate the local stability of the r-th specimen in the set $(\mathrm{r} \in\{1,2, \ldots, \mathrm{n}\})$, a two-steps procedure must be accomplished. The first step deals with the detection of the Direct Matching Points (DMPs) between signature $\mathrm{S}^{r}$ and each other signature $S^{v}$ in the set $(v=1,2, \ldots, n, v \neq r)$. A DMP of the signature $\mathrm{S}^{r}$ with respect to signature $\mathrm{S}^{v}$ is a point which has a direct (one-to-one) coupling with a point of signature $S^{\nu}$ when the two signatures are matched togheter by an elastic matching procedure. More specifically the point $z^{r}(p)$ of the r-th signature coupled with the point $z^{v}(q), q \in\left\{1,2, \ldots, M^{v}\right\}$, is a DMP of the $r$-th signature with respect to the $\mathrm{v}$-th signature if and only if:

and

$$
\forall \mathrm{p}=1,2, \ldots, \mathrm{M}^{r}, \mathrm{p} \neq \mathrm{p}, \mathrm{z}^{r}(\mathrm{p}) \text { is not coupled with } \mathrm{z}^{v} \text { (q) }
$$

$$
\forall \mathrm{q}=1,2, \ldots, \mathrm{M}^{v}, \underline{\mathrm{q}} \neq \mathrm{q}, \mathrm{z}^{v}(\mathrm{q}) \text { is not coupled with } \mathrm{z}^{r}(\mathrm{p})
$$

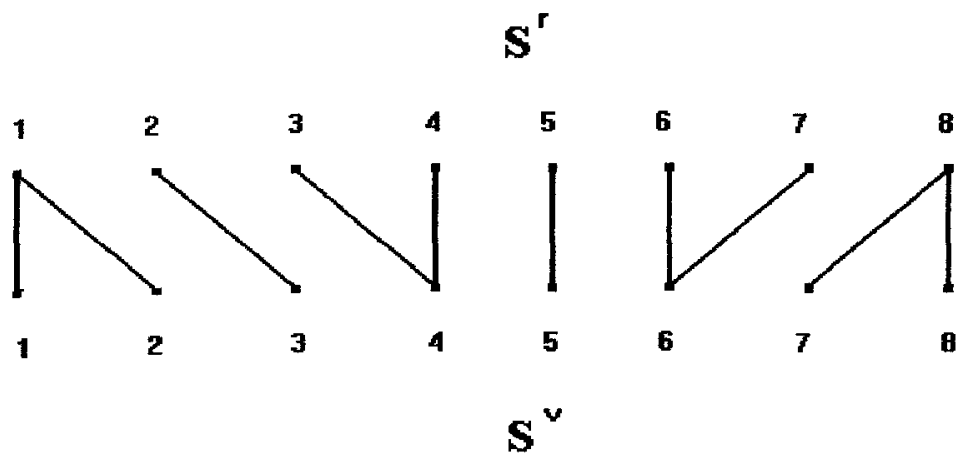

Figure 1: Direct Matching Points 
Figure 1 shows some typical couplings between samples of $\mathrm{S}^{r}$ and $\mathrm{S}^{\nu}$. In this case the DMPs in the sequence $S^{r}$ are the points 2 and 5 . In the second step the local index of stability of the signature $S^{r}$ is computed by a simple avaraging procedure. In fact a DMP indicates the existence of a region of the $r$-th signature which is roughly stable when compared to the corresponding region of the v-th signature. For each point of the r-th signature a score can be introduced according to to its type of coupling with respect to the points of the v-th signture:

$$
\text { Score }^{v}\left(\mathrm{z}^{r}(\mathrm{p})\right)=\left\{\begin{array}{ll}
1 & \text { if } z^{r}(p) \text { is a DMP } \\
0 & \text { otherwise }
\end{array} .\right.
$$

Therefore, the index of local stability of the point $z^{T}(p)$ is given by the frequency of direct matchings of the point when signature $S^{r}$ is matched against the other genuine signatures available:

$$
\mathrm{I}\left(\mathrm{z}^{r}(\mathrm{p})\right)=\frac{1}{n-1} \sum_{\substack{v=1 \\ v \neq r}}^{n} \operatorname{Score}^{\nu}\left(\mathrm{z}^{\gamma}(\mathrm{p})\right)
$$

\section{Selection of the Near-Optimal Set of Reference Signatures}

It is widely accepted that the performance of a signature verification system depends on the characteristics of the set of reference specimens [1]. In this field, beside intrinsic difficulty in imitating a signature, the instability in the set of reference signatures is one of the most common cause of deterioration in accuracy of the verification [1]. However, in many real applications more genuine signatures are available than those required by the system for reference. Therefore, suitable rules are necessary to select the subset of specimens to be used as reference according to some near-optimality criteria. A powerful tool to select such subset is the stability index described previuosly. From the consideration that the improvement of the impostor-acceptance error rate (type II error rate) is generally considered more important than the reduction of the false-rejection error rate (type I error rate), in the following an application of such tool is presented in order to select the subset of signatures for which the importor-acceptance error rate is as lower as possible.

For this purpose, let be $\mathrm{R}^{1}, \mathrm{R}^{2}, \ldots, \mathrm{R}^{n}$ the set of genuine signatures available, $\mathrm{z}^{\mathrm{i}}=\mathrm{R}^{\mathrm{i}}$ for $\mathrm{i}=1,2, \ldots, \mathrm{n}$, and $\mathrm{I}\left(\mathrm{z}^{1}().\right), \mathrm{I}\left(\mathrm{z}^{2}().\right), \ldots, \mathrm{I}\left(\mathrm{z}^{n}().\right)$ the respective indexes of local stability. The selection of the near-optimal subset of $\mathrm{m}$ reference signatures $(m<n)$ is accomplished by selecting specimens which show wide stable regions in corresponding parts of the trace. This criterion descends from the wellknown assumption that the imitation of highly stable pattern in a wide region is more difficult than the imitation of minor, variable patterns. Thus, the near-optimal $\mathrm{m}$-tupla of reference signatures is the $\mathrm{m}$-tupla: 


$$
\mathbf{R}^{\mathbf{i}_{1}}, . ., \mathbf{R}^{\mathbf{i}_{j}}, . . \mathrm{R}^{\mathbf{i}_{\mathrm{m}}}
$$

(where $\forall \mathrm{j}=1,2, \ldots, \mathrm{m}, \mathrm{i} \in\{1,2, \ldots, \mathrm{n}\}$; and $\forall \mathrm{j}, \mathrm{t}=1,2, \ldots, \mathrm{m}, \mathrm{j} \neq \mathrm{t} \Rightarrow \mathrm{i}_{\mathrm{j}} \neq \mathrm{i}_{\mathrm{t}}$ ) for which it follows:

$\underset{m-\text { tuple }}{\text { Max }} \quad i_{j} \quad \sum_{p}\left[\prod_{\substack{a=i_{1}, i_{2}, \ldots, i_{m} \\ a \neq i_{j}}} I_{a}\left(z^{i_{j}}(p)\right)\right]$

where

$$
I_{a}\left(z^{i_{j}}(p)\right)=\frac{1}{\operatorname{card}\left(K_{a}^{i_{j}}(p)\right)} \sum_{q \in K_{a}^{i j}(p)} I\left(z^{a}(q)\right)
$$

and

$$
\mathrm{K}_{\mathrm{a}}^{\mathrm{i}_{\mathrm{j}}}(\mathrm{p})=\left\{\mathrm{q} \mid \text { point } \mathrm{z}^{\mathrm{a}}(\mathrm{q}) \text { is coupled with } \mathrm{z}^{\mathrm{i}_{\mathrm{j}}}(\mathrm{p})\right\}
$$

\section{Experimental Results}

The stability index proposed in this paper has been used to select the nearoptimal subset of reference specimens for the verification system presented in ref. [5].The test has been carried out using a graphic tablet of $500 \mathrm{dpi}$ and $110 \mathrm{~Hz}$, connected to a PS/2 (80486 - 33Mhz). For the experimental test, two databases of online signatures have been used for each of the ten signers considered. The first database contains fifty-seven genuine signatures, the second database contains fifty forgeries written by ten forgers in controlled writing sessions. Each forger has had about ten minutes to practice himself with the electronic tablet and five minutes to affix five signatures.

For each signer, seven genuine signatures have been randomly selected and used as the set of available reference signature. Among this set, the near-optimal subset of three signatures has been selected and used as reference for the verification system. In the test sessions, the standard dissimilarity measure has been considered both for the selection of the reference signatures and for the verification process:

$$
\mathrm{D}\left(S^{r}, S^{\nu}\right)=\sum_{k=1}^{K} d\left(c_{k}\right)
$$


where the measure $d\left(c_{k}\right)$ is defined for each coupling $c_{k}=\left(i_{k}, j_{k}\right), k=1,2, \ldots, K$, provided by the elastic matching procedure. Moreover such measure has been defined with respect to different domains of representation of the signatures:

(1) position

$$
d\left(c_{k}\right)=d\left(z^{r}\left(i_{k}\right), z^{v}\left(j_{k}\right)\right)=\sqrt{\left(x^{r}\left(i_{k}\right)-x^{v}\left(j_{k}\right)\right)^{2}+\left(y^{r}\left(i_{k}\right)-y^{v}\left(j_{k}\right)\right)^{2}}
$$

(2) velocity

$$
d\left(c_{k}\right)=d\left(v^{r}\left(i_{k}\right), v^{v}\left(j_{k}\right)\right)=\sqrt{\left(v_{x}^{r}\left(i_{k}\right)-v_{x}^{v}\left(j_{k}\right)\right)^{2}+\left(v_{y}^{r}\left(i_{k}\right)-v_{y}^{v}\left(j_{k}\right)\right)^{2}}
$$

(3) acceleration

$$
d\left(c_{k}\right)=d\left(a^{r}\left(i_{k}\right), a^{v}\left(j_{k}\right)\right)=\sqrt{\left(a_{x}^{r}\left(i_{k}\right)-a_{x}^{v}\left(j_{k}\right)\right)^{2}+\left(a_{y}^{r}\left(i_{k}\right)-a_{y}^{v}\left(j_{k}\right)\right)^{2}}
$$

where for each specimen the velocity and acceleration functions have been derived by the position signals detected directly by the graphic tabled during the apposition process.

When the subset of reference signatures is selected following the criterion presented in this work, the importor acceptance rate of the system is on average three times lower than using other 3-tuple of genuine signatures for reference. Moreover, the possibility to detect for each writer the representation space in which his own signatures are more stable allows to evaluate in a fast way the domain in which the matching procedure is more profitable for each writer. Specifically, for the set of signers under consideration, it has been shown that the more stable domain for signature representation is the velocity domain ( 5 signers), followed by the acceleration ( 3 signers) and position domain ( 2 signers). Figure 2 and 3 shows respectively a genuine signature and its high/low stability regions detected in the velocity domain.

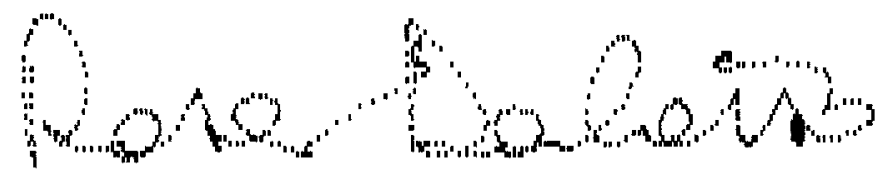

Figure 2. On-line input signature 


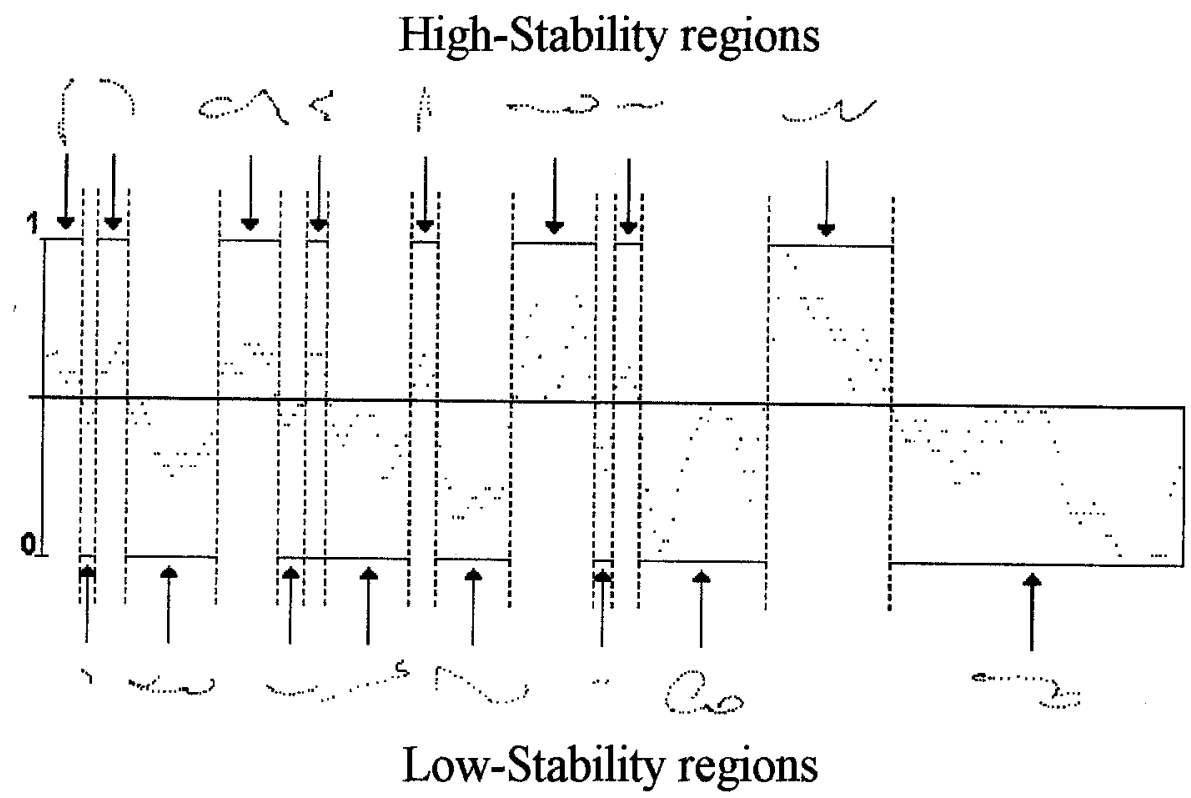

Figure 3. Detection of High/Low stability regions in the velocity domain

\section{Conclusion}

In this paper the local stability in dynamic signatures is measured. Local stability has been applied to select the near-optimal set of reference signatures for online signature verification. The experimental results demonstrate the usefulness of this information for the development of high-accuracy signature verification systems.

\section{References}

[1] G. Pirlo, "Algorithm for Signature Verification", in Fundamentals in Handwriting Recognition, NATO-ASI Series, Springer-Verlag, pp.139-152, 1994.

[2] Progress in Automatic Signature Verification, World Scientific Publ., R. Plamondon (ed.), 1994.

[3] J.-J. Brault, R. Plamondon, "A complexity Measure of Handwritten Curves:. Mideling of Dynamic Signature Forgery", IEEE Transactions on System, Man, and Cybernetics, Vol. 23, no. 2, March/April 1993, pp. 400-413.

[4] G. Congedo, G. Dimauro, S. Impedovo, G. Pirlo, "A New Methodology for the Measurement of Local Stability in Dynamical Signatures", Proc. of the Fourth International Workshop on Frontiers in Handwriting Recognition, Taipei, Taiwan, Dec. 7-9, 1994, pp. 135-144.

[5] G. Dimauro, S. Impedovo, G. Pirlo, " On-line Signature Verification through a Dynamical Segmentation Technique", Proc. of the Third International Workshop on Frontiers in Handwriting Recognition, Buffalo-NY, USA, 1993, pp. 262-271. 